\title{
Acute obstructive suppurative pancreatic ductitis in pancreatic malignancies
}

\section{다 $($ ) $\odot$}

\author{
Authors \\ Ryoko Shimizuguchi, Masataka Kikuyama, Terumi Kamisawa, Sawako Kuruma, Kazuro Chiba
}

Institution

Department of Gastroenterology, Tokyo Metropolitan

Cancer and Infectious Diseases Center Komagome Hospital, Tokyo, Japan

submitted 20.5.2020

accepted after revision 3.9 .2020

Bibliography

Endoscopy International Open 2020; 08: E1765-E1768

DOI 10.1055/a-1268-7086

ISSN 2364-3722

(C) 2020. The Author(s).

This is an open access article published by Thieme under the terms of the Creative Commons Attribution-NonDerivative-NonCommercial License, permitting copying and reproduction so long as the original work is given appropriate credit. Contents may not be used for commecial purposes, or adapted, remixed, transformed or built upon. (https://creativecommons.org/licenses/by-nc-nd/4.0/)

\section{Corresponding author}

Masataka Kikuyama, MD, PhD, Department of Internal

Medicine, Tokyo Metropolitan Komagome Hospital, 3-18-22

Honkomagome, Bunkyo-ku, Tokyo 113-8677, Japan

Fax: +1-81-3-3823-5433

kikuyama110@yahoo.co.jp

\section{ABSTRACT}

Background and study aims Acute obstructive suppurative pancreatic ductitis (AOSPD) is a suppurative pancreatic duct infection with main pancreatic duct (MPD) or accessory pancreatic duct obstruction in the absence of a pancreatic pseudocyst or necrosis, which is experienced usually in chronic pancreatitis. The diagnosis is confirmed by the finding of pancreatic duct obstruction on endoscopic retrograde cholangiopancreatography (ERCP) with evidence of infection, such as a positive pancreatic juice culture or drainage of purulent pancreatic juice.

Patients and methods We studied five patients with pancreatic ductal adenocarcinoma (PDAC) and one with chronic myelogenous leukemia (CML), who suffered from AOSPD. Results of the 281 PDAC and 39 CML patients who we treated in the past 2 years in our hospital, five with PDAC (1.8\%) and one with CML (2.6\%) experienced AOSPD. Each patient had fever, abdominal pain, and increased blood Creactive protein. Pancreatography found that each patient had a MPD stricture and an upstream dilatation. Four had a disruption of the MPD in the upper stream of the stricture. Nasopancreatic drainage was successfully performed in all patients. Pancreatic juice culture was positive for Klebsiella pneumonia, Enterobacter agerogenes, or Enterococcus cloacae in four patients.

Conclusion AOSPD should be considered in pancreatic malignancy with fever and abdominal pain. Prompt diagnosis of AOSPD could avoid shortening of survival of patients with an already poor prognosis by infection.

\section{Introduction}

Acute obstructive suppurative pancreatic ductitis (AOSPD) is a rare pancreatic duct disorder without concomitant pancreatic cyst, abscess, or necrosis [1]. Most cases of AOSPD have occurred in patients with chronic pancreatitis, but a few have been complications of pancreatic cancer [2,3]. The most frequent manifestation of AOSPD is a high fever with abdominal pain, which is also common in chronic pancreatitis and pancreatic cancer. This report describes our experience with six patients with pancreatic malignancies and AOSPD, their clinical manifestations and treatment.

\section{Patients and methods}

We studied five patients with pancreatic ductal adenocarcinoma (PDAC) and one with chronic myelogenous leukemia (CML), in the past 2 years in Tokyo Metropolitan Cancer and Infectious Diseases Center Komagome Hospital. The clinical and radiological records were reviewed retrospectively. Clinical characteristics, presentation, laboratory findings, imaging findings, and treatments were investigated in all six patients. 
- Table 1 Patient characteristics.

\begin{tabular}{|c|c|c|c|c|c|c|c|c|c|c|}
\hline Case & Sex & $\begin{array}{l}\text { Age } \\
\text { (years) }\end{array}$ & $\begin{array}{l}\text { Diag- } \\
\text { nosis }\end{array}$ & $\begin{array}{l}\text { Abdom- } \\
\text { inal pain }\end{array}$ & $\begin{array}{l}\text { Maximum } \\
\text { tempera- } \\
\text { ture }\left({ }^{\circ} \mathrm{C}\right)\end{array}$ & $\begin{array}{l}\text { WBC } \\
(/ \mu \mathrm{L})\end{array}$ & $\begin{array}{l}\text { CRP } \\
\text { (mg/dL) }\end{array}$ & $\begin{array}{l}\text { AMY } \\
\text { (U/I) }\end{array}$ & $\begin{array}{l}\text { MPD di- } \\
\text { ameter } \\
(\mathrm{mm})\end{array}$ & $\begin{array}{l}\text { Pancreatic juice } \\
\text { cultures }\end{array}$ \\
\hline 1 & $\mathrm{~F}$ & 80 & PDAC & + & 39.1 & 7800 & 29.2 & 113 & 12 & Klebsiella pneumoniae \\
\hline 2 & M & 64 & PDAC & + & 39.0 & 13900 & 13.46 & 98 & 9 & Klebsiella pneumoniae \\
\hline 3 & $\mathrm{~F}$ & 86 & PDAC & + & 38.7 & 22800 & 42.48 & 2955 & 4 & Enterococcus cloacae \\
\hline 4 & $\mathrm{~F}$ & 86 & PDAC & + & 39.0 & 13500 & 25.84 & 117 & 10 & None \\
\hline 5 & M & 86 & PDAC & + & 38.4 & 8700 & 21.8 & 1536 & 5 & None \\
\hline 6 & M & 37 & CML & + & 38.2 & 14100 & 25.18 & 546 & 10 & Enterobacter agerogenes \\
\hline
\end{tabular}

\section{Results}

\section{Clinical characteristics and presentation}

Of the 281 patients with PDAC and 39 with CML who we treated in the past 2 years, five with PDAC (1.8\%) and one with CML (2.6\%) experienced AOSPD. Key characteristics of these six patients are detailed in $>$ Table 1 . All six patients were Japanese, and three were male and three female. The mean age of the six patients was 73 years (range $37-86$ years). All six complained of fever with abdominal pain.

\section{Laboratory findings and imaging findings}

In six patients, white blood cell (WBC) count and C-reactive protein (CRP) levels were $7800-22800 / \mu \mathrm{L}$ (median $13700 / \mu \mathrm{L}$ ) and $13.5-42.48 \mathrm{mg} / \mathrm{dL}$ (median 25.5), respectively. Computed tomography (CT) showed a markedly dilated main pancreatic duct (MPD) in four patients. Maximum diameter of MPD was 4 to $12 \mathrm{~mm}$ (median $9.5 \mathrm{~mm}$ ). Pancreatography found strictures of the MPD in the head of the pancreas in five patients and in the body in one patient. All had upstream dilatation of the MPD to various degrees, and four had a disruption of the MPD in the dilated portion. One patient had a previous endoscopic retrograde cholangiopancreatography (ERCP) to diagnose pancreatic cancer 1 month before onset of AOSPD.

\section{Treatments}

All patients underwent ERCP to drain the pancreatic duct. In one patient, a nasopancreatic drainage (NPD) tube placement was tried but failed due to patient body movement during ERCP. In five patients, ERCP showed the strictured part of the MPD was upstream. To drain the dilated MPD, a 5-Fr NPD tube (Cook Medical, Bloomington, Indiana, United States or Olympus, Japan) was placed in the pancreas. The fever and abdominal pain resolved in all patients within a few days of placement of a NPD tube. Removal of the NPD alone increased risk of inducing AOSPD, because the MPD stricture remained due to pancreatic malignancies. In four patients, an NPD was replaced with a pancreatic stent after recognizing repairing of pancreatic duct disruption by pancreatography via the NPD tube to avoid recurrence of AOSPD. Cultures of pancreatic juice obtained from the drainage tubes in four patients were positive results for Klebsiella pneumonia, Enterobacter agerogenes, or Enter- ococcus cloacae. In one patient with a pancreatic duct disruption, it was not possible to obtain a sample of pancreatic juice adequate for culture after placement of NPD. All patients used antibiotics.

\section{Case report}

Patient 2 was a 64-year-old man with stage IIA PDAC. He experienced fever and complained of abdominal pain. The WBC count was $13,900 / \mu \mathrm{L}$ and blood CRP was elevated $(13.46 \mathrm{mg} /$ $\mathrm{dL})$. Computed tomography revealed that the MPD was dilated ( $\triangleright$ Fig. 1), and that the size had increased since the diagnosis of pancreatic cancer ( $\$$ Fig. 2 ). ERCP revealed a stricture of the MPD in the pancreas head and dilation of the MPD upstream of the stricture that contained a pancreatic duct disruption ( $\triangleright$ Fig.3). A 5-Fr NPD tube (Olympus, Japan) was passed through the stricture. The drainage fluid contained pancreatic juice and pus; cultures were positive for $K$. pneumoniae. The patient's symptoms were relieved on Day 3 following treatment and pancreatography through the NPD on day 10 revealed resolution of the dilation and repair of the MPD disruption (> Fig.4).

\section{Discussion}

AOSPD is a suppurative pancreatic duct infection with MPD or accessory pancreatic duct obstruction in the absence of a pancreatic pseudocyst or necrosis [1]. The diagnosis is confirmed by the finding of pancreatic duct obstruction on ERCP with evidence of infection, such as a positive pancreatic juice culture or drainage of purulent pancreatic juice $[2,4,5]$. The pancreatic duct obstruction can be caused by calculi or strictures. Most cases of AOSPD occur in patients with chronic pancreatitis, but a few have been reported in pancreatic cancer patients [ $1,6,7]$. PubMed searches for reports of suppurative pancreatic duct or suppuration of the pancreatic duct identified 29 previous cases [1-3,6-11]. Eleven cases had symptoms of infection and positive pancreatic juice cultures without an infection-related pancreatic cystic lesions or intraductal papillary mucinous neoplasms. Three of the 11 cases had pancreatic cancer $[2,3,6]$ and two developed AOSPD after ERCP $[2,3]$. Only one primary onset AOSPD was a naïve case [6]. Klebsiella species were cultured in four of the 11 cases, E. coli in two, Citrobacter freundii 


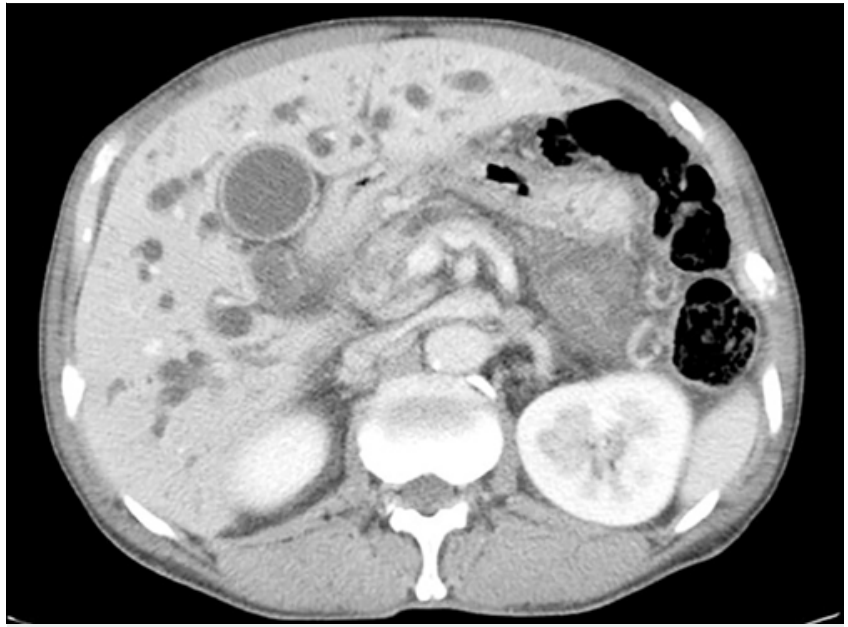

- Fig. 1 Abdominal CT on admission showing a dilated main pancreatic duct (arrow) that was larger than at diagnosis of pancreatic cancer.

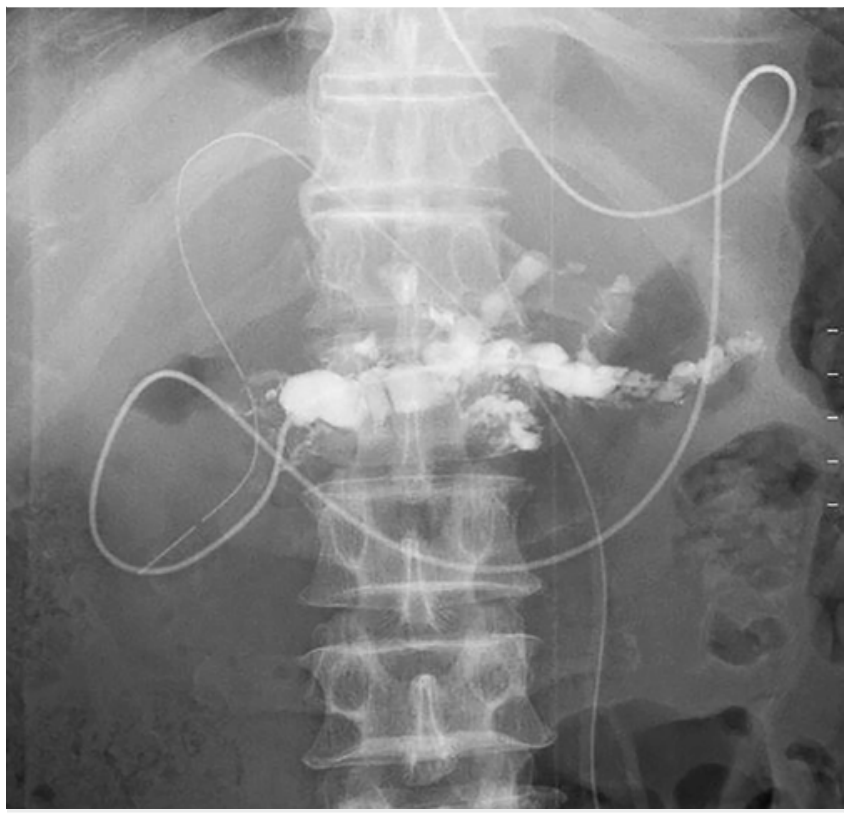

- Fig. 3 Pancreatography through the nasopancreatic drainage tube showing a stricture of the main pancreatic duct (arrowhead) with upstream dilation and extravasation of contrast medium (arrow).

in two, and Enterobacter cloacae, Streptococcus constellatus, Enterococcus faecalis, and Stenotrophomonas maltophilia in one patient each.

The pathogenesis of AOSPD is not clear and there is no standard treatment. Weinman proposed that the effectiveness of the antibacterial agents secreted into the pancreatic juice is decreased during chronic pancreatic juice stasis and bacterial infection is facilitated [1]. We presume that impaired flow of pancreatic juice may compromise the integrity of the intestinal mucosa [11] allowing bacterial translocation from the intestine to the blood or lymph [5], and subsequent movement of the

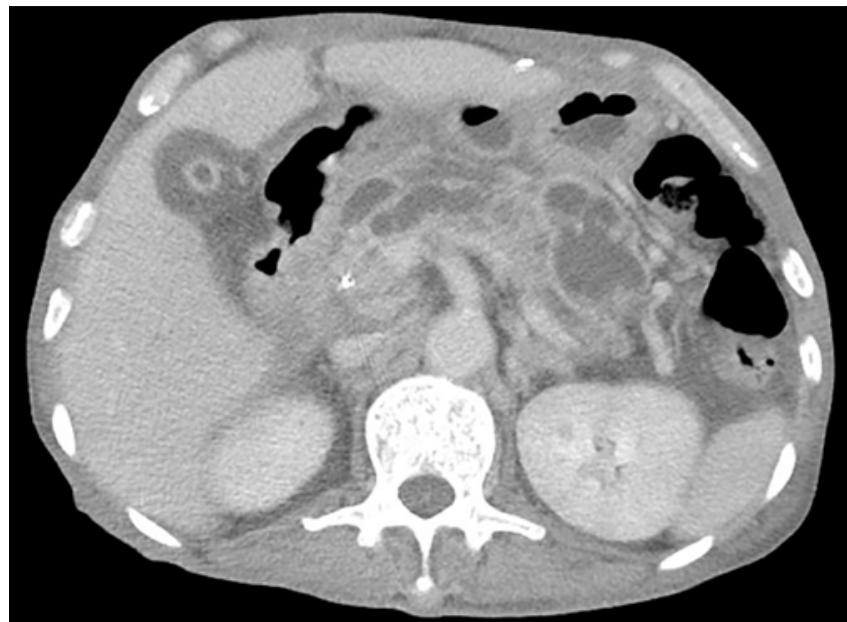

- Fig. 2 Abdominal CT at the time of the diagnosis of pancreatic cancer showing a dilated main pancreatic duct (arrow) and tumor (arrowhead) near the pancreas head.

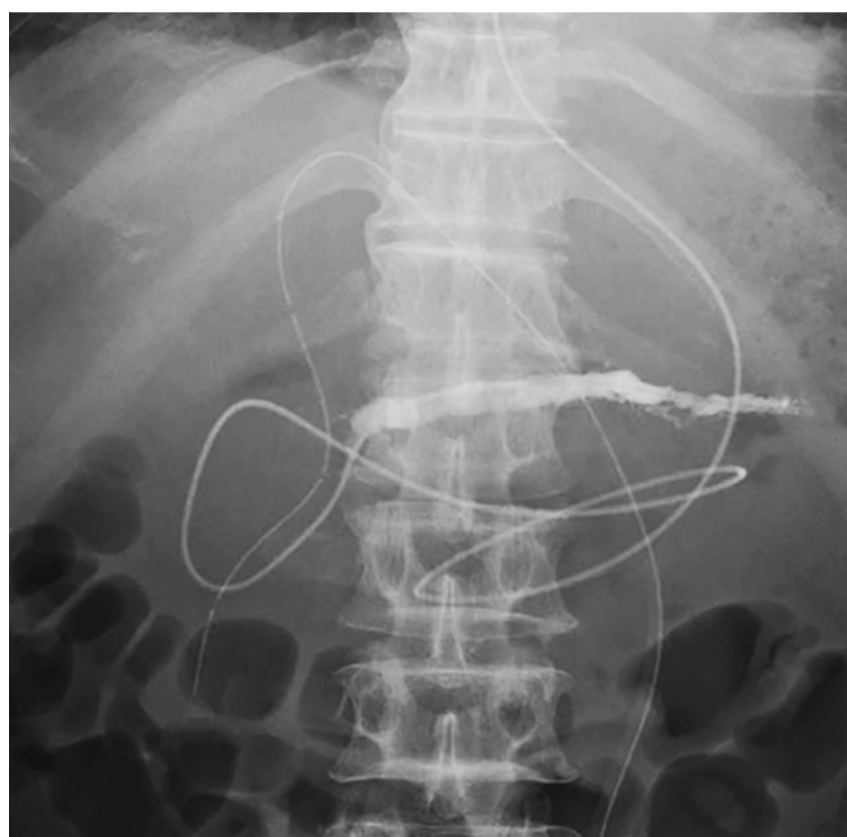

> Fig. 4 Pancreatography through the nasopancreatic drainage tube on Day 10 after treatment showing resolution of pancreatic duct dilation and repair of duct disruption.

circulating bacteria from the bloodstream into the pancreatic duct and overgrowth in the static pancreatic juice might account for the development of AOSPD. The second mechanism is consistent with the culture of bacteria belonging to species found in the intestinal flora $[5,6]$.

Five of the 6 patients with AOSPD had PDAC and one had chronic myelogenous leukemia. As our hospital is a cancer institute, our patients are limited to those with malignancies. A $2 \%$ incidence of AOSPD in patients with malignancies is low but cannot be ignored, because diagnosing and successful treatment avoids the risk of shortening the survival of patients with 
an already poor prognosis by an infection. Each of the patients had characteristic AOSPD symptoms including fever, abdominal pain, and elevated C-reactive protein. Their WBC and the amylase levels probably differed because of the different times of diagnosis and differences in pancreatic exocrine function.

Pancreatography found pancreatic duct disruption in four of six patients. The disruption could have been caused by increased intraductal pressure related to obstruction of pancreatic juice flow, but the action of translocated bacteria could also have been involved. Bacteria produce serine protease, a kind of enzymes, and the enzyme can damage the tight junctions between pancreatic duct epithelial cells and allow leakage of pancreatic juice through protease-activated receptor 2 (PAR2) [12]. The AOSPD resolved with treatment by NPD. A quantity of pancreatic juice sufficient for culture was obtained in four patients, and the K. pneumonia, Enterobacter agerogenes, and Enterococcus cloacae that were isolated are typically found in the intestine, which could secrete serine protease.

NPD and pancreatic stent (PS) have both been used to achieve pancreatic duct drainage. NPD is external, which facilitates obtaining pancreatic juice samples and performing pancreatography as needed [13-15]. The tube is rarely occluded and the integrity of the pancreatic duct is maintained because the tube extends from the pancreatic duct to the exterior of the patient and does not allow duodenal juice containing various bacteria to enter the pancreatic duct. However, the patient must be able to tolerate placement of an NPD tube. Placement of a PS is not a burden to the patient, but it may occlude and induce pancreatitis, and it facilitates entry of duodenal juice into the pancreatic duct, violating its integrity [16]. In patients with pancreatic duct disruption, duodenal juice that enters the pancreatic duct can move to the peripancreatic space, resulting in severe infection. To maintain intraductal integrity and avoid infection resulting from reflux of duodenal juice into the pancreatic duct, NPD is a better choice than PS placement for treating AOSPD.

The outcome of patients with pancreatic malignancy cannot be changed, but AOSPD is an unexpected, treatable event that affects survival and should be treated. AOSPD should be considered when a patient with a pancreatic malignancy develops fever and abdominal pain.

\section{Conclusions}

AOSPD is a rare but treatable condition, which should be considered in pancreatic malignancy with fever and abdominal pain. Diagnosing and treating it could avoid shortening the survival of patients with an already poor prognosis. Pancreatic duct drainage should be considered for treatment, and we recommend NPD for the treatment.

\section{Competing interests}

The authors declare that they have no conflict of interest.

References

[1] Weinman DS. Acute suppuration of the pancreatic duct. Gastrointest Endosc 1995; 41: 268-270

[2] Isono $\mathrm{Y}$, Matsuzaki S, Tanaka $\mathrm{H}$ et al. Acute obstructive suppurative pancreatic ductitis after endoscopic retrograde cholangiopancreatography in a patient with carcinoma of the pancreatic head: a case report. Nihon Shokakibyo Gakkai Zasshi 2016; 113: 289-295

[3] Kondo H, Naitoh I, Okumura F et al. Clinical features of acute obstructive suppurative pancreatic ductitis: A retrospective review of 20 cases. J Gastroenterol Hepatol 2016; 31: 1366-1373

[4] Fujimori N, Igarashi H, Ito T. Acute obstructive suppurative pancreatic ductitis. Clin Gastroenterol Hepatol 2011; 9: 28

[5] Ota Y, Kikuyama M, Kurokami T et al. Six cases of acute obstructive suppurative pancreatic ductitis. The journal of Japan Pancreas Society 2012; 27: 755-761

[6] Tajima $Y$, Kuroki T, Susumu $S$ et al. Acute suppuration of the pancreatic duct associated with pancreatic ductal obstruction due to pancreas carcinoma. Pancreas 2006; 33: 195-197

[7] Deeb L, Bajaj J, Bhargava S et al. Acute suppuration of the pancreatic duct in a patient with tropical pancreatitis. Case Rep Gastroenterol 2008; 2 : 27-32

[8] Fujinaga T, Nishida T, Miyazaki M et al. Acute suppurative pancreatic ductitis associated with pancreatic duct obstruction. Endoscopy 2013; 45: 135

[9] Inoue T, Ito K, Ishii N et al. Acute obstructive suppurative pancreatic ductitis associated with type 1 autoimmune pancreatitis. Pancreas 2017; 46: 24-25

[10] Wali E, Koo P, Packer CD. Acute obstructive suppurative pancreatic ductitis in all asymptomatic patient. Case Rep Med 2015: 919452

[11] Nishie H, Okumura F, Fukusada S et al. A case of intraductal papillary mucinous carcinoma found with acute obstructive suppurative pancreatic ductitis and liver abscess, and associated with a pancreatobiliary fistula. Nihon Shokakibyo Gakkai Zasshi 2013; 110: 1304-1312

[12] Enjoji S, Ohama T, Sato K. Regulation of epithelial cell tight junctions by protease-activated receptor 2. J Vet Med Sci 2014; 76: 1225-1229

[13] Costamagna G, Mutignani M, Ingrosso M et al. Endoscopic treatment of postsurgical external pancreatic fistulas. Endoscopy 2001; 33: 317-322

[14] Miyachi A, Kikuyama M, Matsubayashi Y et al. Successful treatment of pancreaticopleural fistula by nasopancreatic drainage and endoscopic removal of pancreatic duct calculi: A case report. Gastrointest Endosc 2004; 59: 454-457

[15] Rana SS, Bhasin DK, Nanda M et al. Endoscopic transpapillary drainage for external fistulas developing after surgical or radiological pancreatic interventions. J Gastroenterol Hepatol 2010; 25: 1087-1092

[16] Kozarek RA, Ball TJ, Patterson DJ et al. Transpapillary stenting for pancreaticocutaneous fistulas. J Gastrointest Surg 1997; 1: 357-361 